\title{
Variabilidad en la vivencia del parto y su implicación en los cuidados de enfermería
}

\author{
Variability in the experience of labour and its \\ implication in nursing care
}

\section{Variabilidade na experiência do trabalho e sua implicação em cuidados de enfermagem}

\author{
$\mathrm{M}^{\mathrm{a}}$ del Mar Suárez Cadenas ${ }^{1}$, Fernando J. Plaza del Pino ${ }^{2}$ \\ ${ }^{1}$ Enfermera asistencial. Hospital Clínico San Carlos de Madrid.
}

${ }^{2}$ Doctor en Enfermería. Enfermero asistencial. Hospital Torrecárdenas de Almería. Profesor Asociado. Facultad de Ciencias de la Salud. Universidad de Almería.

Cómo citar este artículo en edición digital: Suárez Cadenas, Ma. M., \& Plaza del Pino, F.. (2018). Variabilidad en la vivencia del parto y su implicación en los cuidados de enfermería. Cultura de los Cuidados (Edición digital), 22(50). Recuperado de http://dx.doi.org/10.14198/cuid.2018.50.03

Correo electrónico: mmsuca12@gmail.com

Correspondencia: C/ Vera n 2104007 Almería, España

Recibido: 23/05/2017; Aceptado: 12/10/ 2017

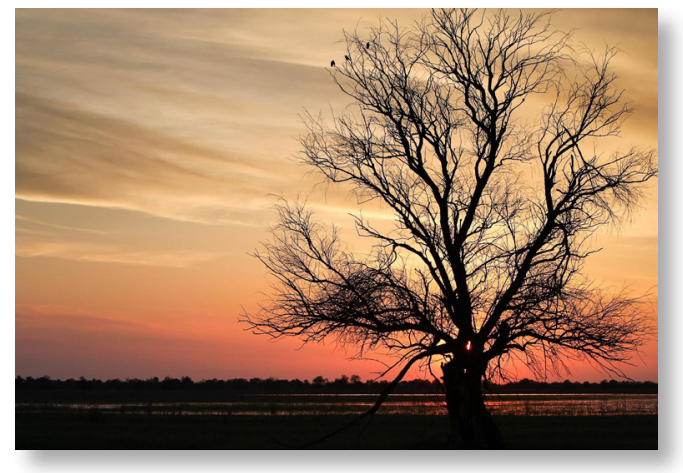

\section{ABSTRACT}

Cultural diversity includes multiple forms of interpreting the experience of pain, from the point of view of nursing we must take interest in the different cultural collectives to better understand their ways of understanding the world with the goal of adapting our cares. In this study, we delve into the variability of the experience of labour in immigrant women. Main objective: Delving into the diversity of the experiences of the process of labour in immigrant women. Methods: Bibliographic review. We used following inclusion criteria articles were accepted since 2000 until now.
Those that were full text were accepted and fit the proposed theme. We found 57 articles of which 31 were selected for the final review. Results: We have organised the different found themes into three block; immigrant women and health, variability in the process of labour and nursing implication. Conclusions: The variability in the experience of labour does not only appear between different cultural groups, but also within the same culture, this variability demands from us an adaptation in the cares we offer to different collectives and regarding the different ways of acting during the process of labour, we have not found any paper that proposes a way to do so, new studies are indispensable that not only theorise about the cultural adaptation of cares, but also centre on how to take them into practice.

Key words: Inmigration, woman, nursing, labour, cares.

\section{RESUMEN}

La diversidad cultural incluye múltiples formas de interpretar la vivencia del dolor, 
desde la enfermería debemos acercarnos a los distintos colectivos culturales para comprender mejor sus formas de entender el mundo con la finalidad de adaptar nuestros cuidados. En este estudio profundizamos en la variabilidad en la vivencia del parto en mujeres inmigrantes. Objetivo principal: Profundizar en la diversidad en la vivencia del proceso del parto en mujeres inmigrantes. Metodología: Revisión bibliográfica. Como criterios de inclusión fueron aceptados artículos desde el año 2000 hasta la actualidad. Se admitieron los que fueran de texto completo y se ajustaran a la temática propuesta. Se encontraron 57 artículos de los cuales se seleccionaron 31 para la revisión final. Resultados: Hemos organizado las distintas temáticas encontradas en tres bloques; mujeres inmigrantes y salud, variabilidad en el proceso de parto e implicación enfermera. Conclusiones: La variabilidad en la vivencia del parto no solo se da entre distintos grupos culturales sino dentro de una misma cultura, esta variabilidad nos demanda una adaptación en los cuidados que ofrecemos a los distintos colectivos y el respeto de las distintas maneras de actuar ante el proceso de parto, no hemos encontrado ningún artículo que proponga la manera de hacerlo, son imprescindibles nuevos estudios que además de teorizar sobre adaptación cultural de los cuidados se centren en cómo llevarlos a la práctica.

Palabras clave: Inmigración, mujer, enfermería, parto, cuidados.

\section{RESUMO}

A diversidade cultural inclui múltiplas formas de interpretação a respeito da existência da dor. Desde a doença devemos nos aproximar dos diversos coletivos culturais para compreender melhor suas fomas de interpretação do mundo com a finalidade de adequar nossas atenções. Nesse estudo aprofundamos as diversas formas de ocorrência do parto em mulheres imigrantes. Objetivo principal: Aprofundar-se na diversidade na vivencia do proceso do parto em mulheres imigrantes. Metodologia: Revisão bibliográfica. Utilizouse como critérios de inclusão artigos de 2000 ao apresente. Eles foram admitidos aqueles que estavam de texto completo e estar de acordo com o tema proposto. Encontrandose 57 artigos, dos quais 31 foram seleccionados para análise final encontrada. Resultados: temos organizado diversas temáticas em três blocos; mulheres imigrantes e saúde, variações no processo de parto, e implicação nas doença. Conclusões: A variabilidade na ocorrência do parto não só se dá entre grupos culturais distintos, como também dentro de uma mesma cultura, sendo que esta variabilidade nos exige adaptarmos aos cuidados que oferecemos aos distintos coletivos e o respeito às diversas maneiras de atuar diante do processo de parto. Não encontramos nenhum artigo que proponha a maneira de fazer-lo, dessa forma, são imprescindíveis novos estudos, que além de teorizar sobre a adaptação cultural em relação aos cuidados, se dediquem a colocá-las em prática.

Palavras chave: Imigração, mulher; doenças, parto, cuidados.

\section{INTRODUCCIÓN}

El fenómeno inmigración es un tema recurrente en la literatura científica en el campo de la salud. Cada cultura tiene una percepción propia sobre qué es enfermedad y con ello sus propias costumbres y cultos a la hora de tratarla, así como de manejar el dolor (Plaza del Pino et al, 2005). La adaptación e individualización consciente ante las diferencias cul- 
turales de nuestros pacientes es necesaria en el campo de la enfermería, buscando un equilibrio entre los diferentes cuidados y costumbres mediante los cuales la persona inmigrante sienta mayor comodidad y familiaridad.

La salud de las mujeres inmigrantes es una cuestión esencial, conocer y concienciarse sobre la adaptación de los cuidados hacia este colectivo no solo beneficia a la propia mujer, sino al ser cuidadora principal, también beneficia a los cuidados de su familia, comunidad, país de asentamiento y país de procedencia (Luque y Oliver, 2005).

Acercarnos a este colectivo es de gran importancia para comprender más sus costumbres y disminuir así los riesgos para la salud que supone el choque cultural a su llegada a España

La percepción y manifestación del dolor es muy importante a la hora de hablar de cuidados transculturales y salud. La diversidad cultural incluye múltiples formas de interpretar la vivencia del dolor, desde la enfermería debemos acercarnos a los distintos colectivos culturales para comprender mejor sus formas de entender el mundo con la finalidad de adaptar nuestros cuidados, diversos autores inciden en esta variabilidad cultural y la necesidad de adaptación de nuestros cuidados (Plaza del Pino et al., 2005; Moreno y Agudo, 2006; Luque y Oliver, 2005). El parto es uno de los procesos fisiológicos en el que más se sufre, tal como revela el estudio realizado por Núñez (2014) en el que muestra la importancia de la mejora en la atención enfocada al sufrimiento del dolor para las parturientas.

El proceso de parto es una experiencia individual y personal en cada mujer. Abarcar los cuidados y entender a cada mujer en su situación, sin juzgarla por su vivencia, colaborará a la hora de criar después a su hijo y cuidar a su familia, a la hora de recurrir a los recursos sanitarios posteriormente e influir en la población. Con este estudio pretendemos conocer la diversidad de la vivencia del parto de nuestras pacientes inmigrantes y las implicaciones que esta variabilidad puede tener en su cuidado.

\section{OBJETIVOS}

Objetivo principal: Profundizar en la diversidad en la vivencia del proceso del parto en mujeres inmigrantes.Objetivos específicos:

- Indagar en la diversidad cultural en la percepción y expresión del dolor en el proceso el parto en mujeres inmigrantes.

- Conocer la implicación que en la práctica enfermera tiene la diversidad cultural en el proceso del parto.

\section{METODOLOGÍA}

Para alcanzar los objetivos de nuestro estudio hemos realizado una revisión bibliográfica distintas bases de datos como Pubmed, Cuiden Plus, Cinahl Complete, Dialnet o Google Scholar. Se introdujeron las siguientes palabras clave: "inmigration", "pain", "health" "givebirth"o su traducción al castellano si la base de datos así lo requería. Los operadores booleanos utilizados fueron: "AND” y "OR".

Criterios de inclusión/exclusión: se aceptaron artículos desde el año 2000 hasta la actualidad. Se admitieron los que fueran de texto completo y se ajustaran a la temática propuesta. Se encontraron 57 artículos de los cuales se seleccionaron 31 para la revisión final.

\section{RESULTADOS}

De los 31 artículos seleccionados 11 tratan sobre la inmigración y salud, 1 sobre la salud reproductiva y la crisis económica, 7 sobre 


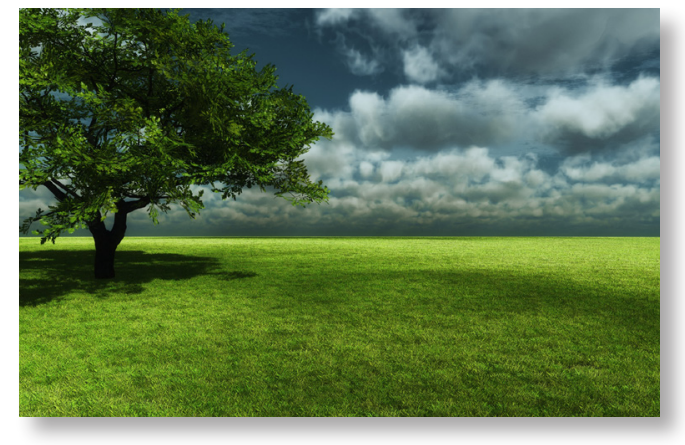

cuidados en mujeres inmigrantes y 12 sobre la percepción y parto para referenciar la mejora de la calidad asistencial.

\section{Agrupación de las temáticas encontradas. Mujeres inmigrantes $y$ salud.}

Tras España convertirse hace varios años en un país lleno de nuevas demandas multiculturales, en lo que todos los artículos coinciden, nos encontramos en la actualidad con un proceso de cambio en esta inmigración, la feminización de los movimientos migratorios (García et al, 2009; Fajardo y Pérez, 2006; Gentil, 2009). El cambio en la composición de género del colectivo de inmigrante modifica a su vez las intervenciones sanitarias priorizadas por los mismos, así, encontramos que el servicio sanitario más utilizado por la población inmigrante es el de obstetricia y ginecología (Moreno, 2008; Fernández, 2006) debido a que la mayoría de mujeres inmigrantes son jóvenes y en edad fértil, además tienen más pariedad tal como refiere uno de los resultados del estudio comparativo entre mujeres nativas e inmigrantes de López-Olmos (2011). El factor primordial para una buena atención en la salud materno-infantil de las mujeres inmigrantes es la inclusión social (Gagnon et al, 2013).

Gentil (2009) muestra en su estudio que no hay un patrón uniforme a todos los inmigrantes, identificando los sentimientos de las mujeres inmigrantes tras una larga experi- encia lejos de su país. De acuerdo a esta diferencia entre culturas y dentro de las mismas, observamos que el seguimiento correcto de los controles sanitarios durante el embarazo defieren en los grupos de mujeres inmigrantes según su lugar de origen (Marín et al, 2008; Valverde et al, 2013; Lajara-Almendros et al, 2011), siendo las mujeres latinoamericanas y de Europa del Este las que más seguimiento correcto tienen del embarazo a diferencia de las poblaciones magrebíes, subsaharianas y asiáticas con un bajo porcentaje de participación, lo que debe hacer prioritario para los servicios sanitarios la mejora en la captación de los programas de salud materno-infantil de estos colectivos de mujeres.

Goberna et al (2005) analizó a través de las matronas de atención primaria las dificultades que se encuentra la población magrebí para un buen seguimiento del embarazo; el desconocimiento del idioma para la educación sanitaria y entrevistas, la falta de material de soporte específico en educación sanitaria para personas inmigrantes y mediadores culturales, y la importancia de la experiencia previa en interculturalidad del profesional, concluye con la necesidad de potenciar las medidas de mejora de la comunicación y conocimiento de la diversidad e interculturalidad de los profesionales sanitarios.

En cuanto al perfil epidemiológico en mujeres inmigrantes respecto a partos de mayor riesgo, su proporción es similar al de las mujeres autóctonas, una diferencia clara es que las mujeres inmigrantes paren más jóvenes y utilizan menos la anestesia epidural que las autóctonas (García et al, 2008). En cuanto al porcentaje de cesáreas practicadas a mujeres inmigrantes, existe una mayor incidencia en mujeres latinoamericanas que en el resto de procedencias, lo que se relaciona a la fa- 
cilidad cultural y lingüística de las mujeres procedentes de América del Sur y concluyen con la necesidad de promover el parto vaginal en las mismas, y facilitar el acceso al resto de mujeres procedentes de países del Magreb o la Europa del Este que se encuentran con múltiples barreras para acceder a una cesárea por indicación médica y así disminuir el porcentaje de muerte fetal en estas últimas que es más alto que en las primeras (Río et al, 2010).

En cuanto a la calidad asistencial percibida Lajara-Almendros et al (2011) afirma que las mujeres inmigrantes presentan mayor grado de satisfacción que las nativas, sugiriéndonos que las expectativas de la paciente inmigrante pudieran ser menores que la de la autóctona. Refiere así la necesidad de controles periódicos de la unidad de Calidad del servicio de Salud y la mejora de la captación precoz de las mujeres inmigrantes embarazadas desde la atención primaria, aumentando la motivación para que continúen en los programas.

\section{Variabilidad en el proceso de parto.}

Las manifestaciones, expresiones y costumbres con respecto a la salud y enfermedad que cambian de una cultura a otra, incluso dentro del mismo sistema cultural. Luque y Oliver en su estudio de 2005 describen las diferencias en la percepción y vivencia del parto entre mujeres inmigrantes, diferencias en relación al sentido del pudor, a ser atendidas por un profesional varón o a prescindir del acompañamiento de su pareja, a la percepción del dolor, refiriendo que las mujeres de Europa del Este y África subsahariana son mas estoicas y sus movimientos corporales son mínimos y hacen sonidos o cantos culturales, frente a otras culturas en las que se insiste en que la mujer de parto permanezca en silencio. No es posible realizar generalizaciones, con- cluyen, ya que la variabilidad individual es una característica fundamental, aunque esta variabilidad en la asistencia al parto desde el punto de vista temporal, geográfico y en práctica clínica debería reducirse a través de una guía de práctica clínica para unificar criterios (Salgado Barreira et al, 2010).

La experiencia del dolor en el proceso del parto está moldeada por factores diversos tanto de la vida de cada persona como por factores externos, algunos autores proponen que hay que incidir en la mejora en la atención del parto y en las formas de relacionarse e interactuar con la parturienta con la intención de disminuir el sufrimiento del mismo. La atención al parto requiere preparación obstétrica y ginecológica enfermera, pero también antropológica y multicultural (Nuñez, 2014; Aguilera, 2008)

Para acercarnos a la variabilidad cultural en la experiencia del parto es importante conocer las experiencias concretas de mujeres de distintos orígenes culturales y geográficos, encontramos diferentes estudios en esta dirección como el de Aguilera (2008) sobre la experiencia del parto de una mujer boliviana o el de Orte González (2013) centrado en una mujer marroquí.

- En el primero de los estudios Aguilera destaca que la mujer valora la atención recibida en el parto en comparación con la recibida en su país, sentía satisfacción. Otro aspecto de interés es que en los inicios la parturienta prefería cesárea, pero al pensar en la incorporación al trabajo acabo prefiriendo el parto vaginal.

- En el caso analizado por Orte González se destaca que la satisfacción de la usuaria fue buena, ante el dolor del parto encuentra que la mujer árabe no percibe diferencia en el hecho del dolor con nuestra cultura, pero si en- 
cuentra que la dificultad lingüística y la falta de apoyo emocional le pueden predisponer a una peor tolerancia ante el mismo. Ante la administración de epidural no encuentra ningún aspecto negativo ni religioso sobre el que estas mujeres puedan tener prejuicio, pero sí que prefieren evitar el dolor de espalda posterior. Es importante resaltar el valor que las mujeres de tradición musulmana dan al acompañamiento de la familia durante el parto, en Marruecos, por ejemplo, todas las mujeres de la casa quedan con la parturienta para transmitirle el apoyo necesario.

\section{Implicación enfermera.}

Del Rey y Gálvez (2004) presentaron en un estudio los condicionantes ante las diferencias entre las mujeres inmigrantes y nativas son los determinantes de salud de cada población así como los estilos de vida, situación laboral y económica, situación social y cultura sanitaria, entre otras; pero que en cuanto a la asistencia de la mujer gestante o parturienta no existen diferencias con la mujer nativa ya que debe hacerse desde una sensibilidad individual sin que condicione la práctica el lugar de origen de la mujer. Muestra en sus resultados que no se percibe un choque cultural por parte de la población estudiada.

La mayoría de estudios revisados (Plaza del Pino et al, 2005; Castillo et al, 2001; Fernández, 2006) afirman la necesidad de cambio de respuesta de los profesionales sanitarios ante el aumento en la diversidad cultural de la población a la que cuidar, diversidad que se traslada al significado y la vivencia del proceso salud-enfermedad, del dolor, en cuanto a las prioridades ante la salud, recursos a los que recurrir y repercusión la salud y la enfermedad tiene en el entorno de la persona y su familia.
Son muchos los autores que defienden la necesidad de un cambio en la formación de los profesionales sanitarios y una adaptación cultural individualizada en los cuidados que se ofrecen (Gentil, 2006; Moreno Preciado y Agudo Gisbert; 2006). Plaza del Pino y Martínez Gálvez (2012) inciden en su estudio sobre las actitudes de los profesionales de enfermería ante el colectivo inmigrante en la falta de conocimiento ante los nuevos cuidados y concluyen en la necesidad de un esfuerzo del Sistema Sanitario Público ante la nueva realidad social que se está presentando.

Algunos estudios destacan que un elevado porcentaje de profesionales sanitarios creen que las minorías étnicas pueden ser fuente de conflictividad social y relacionan la inmigración con la delincuencia (Plaza del Pino, 2012) lo que nos obliga a formar en atención a la diversidad cultural a los profesionales y dotarnos de recursos para adquirir sensibilidad y conciencia ante los diversos colectivos, formación en cuidados transculturales a través de cursos y adquisición de títulos que ya está implantada en otros países, mientras que en España hay un déficit importante en esta formación específica (Plaza del Pino y Soriano, 2009). El estudio de Pardo Moreno et al (2006) también reclama la necesidad de la mejora de los programas de salud; en su estudio pretende conocer las características sociosanitarias de las mujeres inmigrantes subsaharianas, encontrándose con que la mayoría de la muestra estudiada se encuentra con trabajo sin contrato y situación administrativa irregular.

\section{CONCLUSIONES}

Las conclusiones a las que llegamos según los resultados obtenidos con este estudio son las siguientes: 
El conocimiento de la diversidad cultural es fundamental para poder ofrecer cuidados de calidad en una sociedad cada vez más diversa como es la nuestra. A través del cuidado durante el proceso del parto, podremos acercarnos a las distintas maneras de cuidar y de vivir el dolor en el proceso del parto en mujeres de distintas culturas y la comprensión hacia "lo diferente".

Puesto que observamos que la variabilidad no es sólo cuestión de inmigración sino de diferentes colectivos, tanto dentro de las mismas culturas como de distintas culturas, creemos que también es necesaria esa variabilidad en los cuidados que ofrecemos a los distintos colectivos y el respeto de las distintas maneras de actuar ante el proceso de parto.

Pese a ser la asistencia obstétrica y ginecológica la más demandada por la población inmigrante y repetirse en varios estudios la necesidad de cambio y adaptación cultural en los cuidados no hemos encontrado ningún artículo que proponga la manera de hacerlo.

Destacamos el hecho de no haber encontrado apenas bibliografía científica que se centre exclusivamente en la mujer inmigrante y el proceso de parto, por tanto, en la percepción y expresión de las características de cómo es el dolor, no pudiendo dar respuesta a las características principales de nuestros objetivos.

La totalidad de los estudios revisados inciden en la necesidad de formación específica y continuada para los profesionales de enfermería, además de campañas de sensibilización para la sociedad. La multitud de estudios concluyen que la nueva realidad de nuestro entorno exige un aumento en la formación cultural de nuestros profesionales de la salud.

Como profesionales de la salud debemos adaptarnos a cada paciente e individualizar nuestros cuidados, más aún cuando cuidamos a mujeres en el proceso del parto. En el caso de mujeres extranjeras, este proceso está cargado de su herencia cultural que se reflejará en también en su expresión del dolor, no podemos pretender que nuestras pacientes se ajusten a lo que esperamos de ellas, al contrario, tendremos que asumir que trabajamos con personas diferentes a nosotros en situaciones de sufrimiento y dolor, en este caso ante el proceso de parto, que confían en nosotros y a las que acompañamos. Tendremos que estar preparados para adaptarnos y respetar la multitud de respuestas y actitudes diferentes que podemos encontrarnos en la multitud de pacientes de distintos orígenes culturales y geográficos que atenderemos. Así pues, sensibilizarnos ante la diversidad cultural de nuestras pacientes es esencial para llevar a cabo unos cuidados culturalmente competentes, personalizados y profesionales teniendo como base la comprensión, más aún cuando la persona a la que cuidamos puede no entender nuestro idioma ni nuestra cultura y desconoce el entorno en el que vivirá un momento tan estresante como importante en su vida como lo es el parto.

Es imprescindible la realización de nuevos estudios que se ocupen de esta preocupación que en común tienen diversos autores. Concluimos constatando que sería de gran interés estudios acerca de cómo formar a los profesionales sanitarios de forma dinámica, prestando especial atención a los cuidados en el proceso del parto a mujeres inmigrantes.

\section{BIBLIOGRAFIA}

- Aguilera, F.J. (2008). La vivencia del parto en una extranjera. Arch Memoria. Recuperado de http://0-www. indexf.com.almirez.ual.es/memoria/5/a10807.php Consultado el 07/03/2015

- Aja, E., \& Arango, J. (2006). Veinte años de inmigración en España: perspectivas jurídica y sociológica (1985- 
2004). Barcelona: Bellaterra SA Ediciones.

- Barreira AS, Rozas MM, Ratón ML, Merino GA. (2010). Variabilidad del parto en España. Análisis del conjunto mínimo básico de datos al alta hospitalaria. ProgObstetGinecol, 53(6), 215-222.

- Castillo S., Mazarrasa, L,, \& Sanz B. (2001). Mujeres inmigrantes hablando de su salud. Utilización de sus discursos como estrategia para promocionar su salud. Index de Enferm (edición digital), 34. Recuperado de http:// www.index-f.com/index-enfermeria/34resvista/34_articulo_9-14.php Consultado el 09/02/2015

- Del Rey Márquez, M.D,, \& Gálvez Toro, A. (2004). Mitos vs vivencias de las mujeres inmigrantes: el embarazo, el parto y el puerperio. Una enfermería transcultural. Evidentia, 1(3). Recuperado de http://www.index-f.com/ evidentia/n3/82articulo.php [ISSN: 1697-638X]

- Fernández Molina, M.A. (2006). Fenómenos migratorios y su influencia en los cuidados transculturales en el marco hospitalario. Cultura de los cuidados, 10(20), 62-68. Recuperado de https://rua.ua.es/dspace/bitstream/10045/865/1/culturacuidados_20_8.pdf

- Gagnon, A.J., Carnevale, F., Mehta P,, \& Rousseau H, Stewart D. (2013). Developing population interventions with migrant women for maternal-child health: a focused ethnography. BMC PublicHealth, 13, 471.

- García Ballesteros, A., Jiménez Basco, B., \& Redondo González A. (2009). La inmigración latinoamericana en España en el siglo XXI. Investigaciones Geográficas, Boletín del Instituto de Geografía, UNAM. 70, 55-70.

- García-García, J., Pardo Serrano, C., Hernández Martínez, A., Lorenzo Díaz, M., \& Gil González, D. (2008). Diferencias obstétricas y neonatales entre mujeres autóctonas e inmigrantes. ProgObstetGinecol, 51(2), 53-62.

- García, J.L. (2015). El discurso de la Unión Europea sobre medidas de integración de inmigrantes y sus derivaciones en España. Migraciones Internacionales, 8(1), 127-158

- Gentil, I. (2006). Enfermería y personas de otras culturas. Cultura de los cuidados, 10(19), 37-40. Recuperado en https://rua.ua.es/dspace/bitstream/10045/965/1/culturacuidados_19_05.pdf

- Gentil, I. (2009). Salud y mujeres inmigrantes latinoa- mericanas. Autoestima y resiliencia. Index de Enferm (edición digital), 18(4). Recuperado de http://www. index-f.com/index-enfermeria/v18n4/6995.php Consultado el 12/11/2015.

- Goberna Tricas, J., Viñas Llebot, H., Palacio Tauste, A., Galí García, M., Paulí Cabezas, A., \& Gómez Moreno, C. (2005). Atención al embarazo en mujeres africanas inmigrantes. Percepción de las matronas de asistencia primaria. Enfermería Clínica, 15(2), 88-94.

- Lajara-Almendros, J.M., García Ruíz, M.L., Tébar Morales, J.P., Molina Sánchez, M.P., \& Milán Callado, A.S. (2012). ¿Cómo perciben las puérperas Inmigrantes la atención recibida en el servicio de Obstetricia del complejo hospitalario de Albacete? EnfermClín, 22(2), 76-82.

- Larrañaga, I., Martín, U., \& Bacigalupe, A. (2014). Salud sexual y reproductiva, y crisis económica en España. Informe SESPAS 2014. GacSanit, 28(S1), 109-115.

- Luque Fernández, M.A., \& Oliver Reche, M.I. (2005). Diferencias culturales en la percepción y vivencia del parto. El caso de las mujeres inmigradas. IndexEnferm, 48(49), 9-13.

- López-Olmos, J. (2013). Mujeres inmigrantes versus autóctonas. Diferencias en disfunciones sexuales, infecciones vaginales y lesiones cervicales. ClinInvest Gin Obst, 40(6), 242-252.

- Marín Jiménez, M.N., Morales Romero, A., \& Quirante Carrero, N. (2008). La salud reproductiva en la mujer inmigrante: diferencias entre culturas. Enfermería Docente, $89,10-14$

- Moreno Preciado, M. (2006). Las implicaciones familiares en los cuidados de los Inmigrantes. Cultura de los Cuidados, 12(24), 59-65. Recuperado de https://rua. ua.es/dspace/bitstream/10045/9871/1/CC_24_10.pdf

- Moreno Preciado, M., \& Agudo Gisbert, E. (2006). Formación y Transculturalidad: El cuidado del paciente inmigrado desde la perspectiva del estudiante de enfermería de primer año. Cultura de los Cuidados, 10(20), 55-61. Recuperado de https://rua.ua.es/dspace/bitstream/10045/864/1/culturacuidados_20_7.pdf

- Nuñez, P. (2014). Experiencias en torno al dolor en el parto hospitalario: una aproximación antropológica. Matronas Prof, 15(2), 43-49 


\section{ᄃultura de las Cuidados}

- Orte González, E.M. (2013). La mujer árabe ante la experiencia del parto. Arch Memoria, 10 fasc. 4. Recuperado de http://www.index-f.com/memoria/10/10410.php Consultado el: 5/12/2015.

- Pardo Moreno, G., Engel Gómez, J.L., \& Agudo Polo S. (2006). Acercamiento a las características sociosanitarias de las mujeres subsaharianas de 2 programas de atención a inmigrantes de Madrid. Enferm Clin, 16(5), 264-267.

- Pareja, M.V., Zurita, A.O., Márquez, A.M., Mateos, A.R., Franco, I.J., De la Ossa, R.M., \& Carvajal, M. (2015). Atención en el parto en la mujer inmigrante; características sociodemográficas y de comportamiento en función de la procedencia de la paciente. ClinInvest Gin Obst, 42(2), 50-55.

- Plaza del Pino, F.J., Plaza del Pino, M.D., \& Martínez Gálvez, M.L. (2005). Inmigración en el poniente Almeriense: pobreza, desarraigo, salud mental e implicación en el cuidado. Presencia,1(2). Recuperado de: http:// www.index-f.com/presencia/n2/artículo.php Consultado el 6/12/2015.

- Plaza del Pino, F.J,, Martínez Gálvez, L. (2012). Actitud de la Enfermería ante los derechos sociales de los inmigrantes. Evidentia, 9(39). Recuperado de http://www. index-f.com/evidentia/n39/ev3900.php Consultado el $6 / 02 / 2015$

- Plaza del Pino, F.J. (2012). Prejuicios de las enfermeras hacia la población inmigrante: una mirada desde el Sur de España. Enfermería Global, 27, 87-96.

- Plaza del Pino, F.J. (2011). Ante la diversidad cultural construyamos una enfermería intercultural. Enferm Comun, 7 (1). Recuperado de http://www.index-f.com/es/ comunitaria/v7n1/ec7102.php Consultado el 3/03/2015

- Plaza del Pino, F.J., \& Soriano Ayala, E. (2009). Formación de los profesionales de enfermería: Cuidar en la sociedad multicultural del siglo XXI. Index de Enferm, 18(3), 190-194

- Río, I., et al. (2010). Caesarean section rates in immigrant and native women in Spain: the importance of geographical origin and type of hospital for delivery. European Journeal of Public Health, 20(5), 524-529.

- Recuerda Solana, C., Riquelme Raya, R., Gutiérrez Martínez, M.M., Martínez Gámez, M.M., Hidalgo Ruzzante, N. (2012). Evaluación de medios de comunicación alternativos con mujeres inmigrantes de habla no hispana en partos. Fundación Index. Biblioteca Lascasas, 8(1).

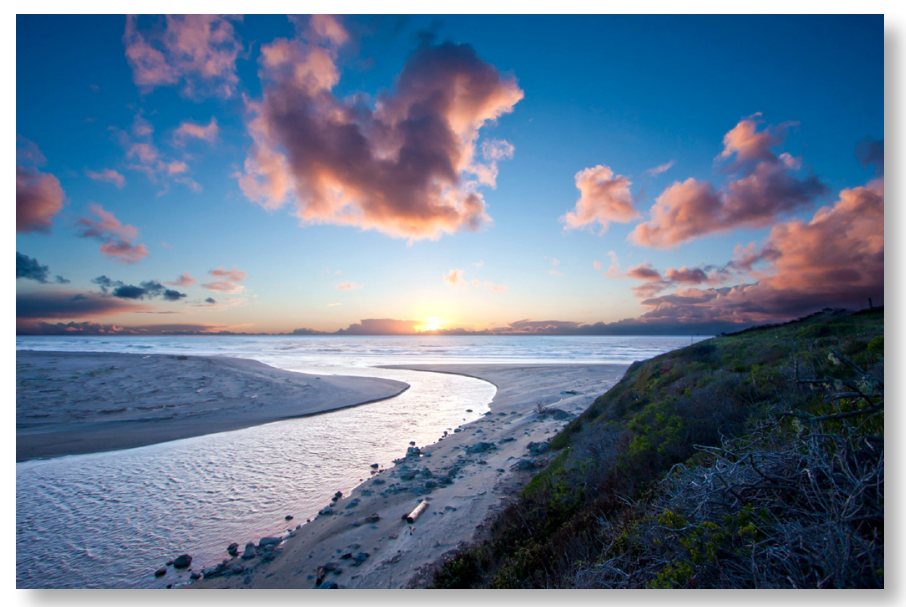

